\title{
Abordagem clínica, diagnóstica e terapêutica da doença de Caroli: uma revisão bibliográfica
}

\author{
Clinical, diagnostic and therapeutic approach to Caroli's disease: a literature review \\ Enfoque clínico, diagnóstico y terapéutico de la enfermedad de Caroli: una revisión de la \\ literatura
}

Isabella Ferraz Ferreira ${ }^{1 *}$, Adriana Rodrigues Ferraz ${ }^{1}$, Alice de Faria Guimarães ${ }^{2}$, Amanda Rodrigues Rios $^{3}$, Ana Luiza Ferreira de Azevedo ${ }^{4}$, Anne Caroline Costa da Silva Carmo ${ }^{5}$, Gabriela Horst Dutra Coutinho ${ }^{6}$, Luiza Thamiris de Oliveira Machado ${ }^{7}$, Natália Parreira Arantes ${ }^{1}$, Tainara Sales Miranda ${ }^{3}$.

\section{RESUMO}

Objetivo: Este artigo buscou reunir, avaliar e sintetizar estudos acerca dos sintomas e dificuldades terapêuticas de pacientes com Doença de Caroli (DC), bem como elucidar o diagnóstico e quais são os possíveis tratamentos definitivos. Métodos: Revisão de 20 publicações científicas indexadas nas seguintes bases eletrônicas de dados: PubMed, SciELO e Science Direct. As publicações revisadas abordam artigos dos últimos 10 anos de natureza metodológica descritiva e qualitativa. Resultados: $O$ diagnóstico da DC consiste em demonstrar a comunicação entre os segmentos dilatados de ductos biliares intrahepáticos e do restante do sistema biliar. É imprescindível uma história clínica detalhada, exame físico preciso e confirmação do exame de imagem, que pode ser realizado por ultrassom, onde se observa a dilatação dos ductos biliares intra-hepáticos, tomografia computadorizada, observando-se o sinal do "ponto central" com imagem de dilatação cística ou por colangiopancreatografia por ressonância magnética, sendo essa última altamente específica para DC. Considerações Finais: Apesar de rara, essa patologia afeta significativamente a qualidade de vida do paciente, devido às repercussões sistêmicas advindas de quadros infecciosos e de suas complicações. Devido a sua gravidade, o diagnóstico precoce se torna crucial, sendo, portanto, fundamental o conhecimento do profissional da saúde acerca dos sinais e sintomas dessa patologia.

Palavras-chave: Doença de Caroli, Ductos biliares, Doenças raras.

\section{ABSTRACT}

Objective: This article aimed to gather, evaluate and synthesize studies on the symptoms and therapeutic difficulties of patients with Caroli Disease (CD), as well as to elucidate the diagnosis and what are the possible definitive treatments. Methods: Review of 20 scientific publications indexed in the following electronic databases: PubMed, SciELO and Science direct. The revised publications address articles from the last 10 years of a descriptive and qualitative methodological nature. Results: The diagnosis of CD consists in demonstrating the communication between the dilated segments of intrahepatic bile ducts and the rest of the bile system. A detailed clinical history, precise physical examination and confirmation of the imaging exam, which can be performed by ultrasound, where the dilation of the intrahepatic bile ducts is observed, computed tomography, observing the "central point" sign with cystic dilation imaging or by cholangiopancreatography by magnetic resonance imaging, the latter being highly specific for CD. Final Considerations: Although rare, this pathology significantly affects the quality of life of the patient, due to the systemic repercussions arising from infectious diseases and their complications. Due to its severity, early diagnosis becomes crucial, and therefore the knowledge of the health professional about the signs and symptoms of this pathology is fundamental.

Keywords: Caroli disease, Bile ducts, Rare diseases.

\footnotetext{
${ }^{1}$ Universidade de Vassouras (UV), Vassouras - RJ. *E-mail: ferrazisabella@hotmail.com

${ }^{2}$ Centro Universitário de Belo Horizonte (UniBH), Belo Horizonte - MG.

${ }^{3}$ Centro Universitário de Caratinga (UNEC), Caratinga - MG.

${ }^{4}$ Faculdade de Minas (Faminas BH), Belo Horizonte - MG.

${ }^{5}$ Centro Universitário Atenas (UniAtenas), Paracatu - MG.

${ }^{6}$ Centro Universitário Redentor (UniRedentor), Itaperuna - RJ.

${ }^{7}$ Universidade Federal de Minas Gerais (UFMG), Belo Horizonte - MG.
} 


\section{RESUMEN}

Objetivo: Este artículo quiso reunir, evaluar y sintetizar estudios sobre los síntomas y dificultades terapéuticas de los pacientes con Enfermedad de Caroli (EC), así como dilucidar el diagnóstico y los posibles tratamientos definitivos. Métodos: Revisión de 20 publicaciones científicas indexadas en las siguientes bases de datos electrónicas: PubMed, SciELO y Science direct. Las publicaciones revisadas abordan artículos de los últimos 10 años de carácter metodológico descriptivo y cualitativo. Resultados: El diagnóstico de la EC consiste en demostrar la comunicación entre los segmentos dilatados de los conductos biliares intrahepáticos y el resto del sistema biliar. Es esencial una historia clínica detallada, un examen físico preciso y la confirmación de las imágenes, que puede realizarse mediante ecografía, donde se observa la dilatación de los conductos biliares intrahepáticos, tomografía computarizada, observando el signo del "punto central" con imágenes de dilatación quística o mediante colangiopancreatografía por resonancia magnética, esta última muy específica para la EC. Consideraciones finales: Aunque es poco frecuente, esta patología afecta la calidad de vida del paciente, debido a las repercusiones sistémicas derivadas de las enfermedades infecciosas y sus complicaciones. Debido a su gravedad, el diagnóstico temprano se vuelve crucial, y por lo tanto el conocimiento del profesional de la salud sobre los signos y síntomas de esta patología es fundamental.

Palabras clave: Enfermedad de Caroli, Conductos biliares, Enfermedades raras.

\section{INTRODUÇÃO}

A Doença de Caroli (DC) é uma patologia rara descrita em 1958 por Jacques Caroli, que a definiu como dilatação sacular multifocal dos ductos biliares intra-hepáticos maiores. Apresenta ocorrência estimada em 1 a cada 10.000 nascidos vivos, com maior incidência em adultos jovens menores de 30 anos (UMAR J, et al., 2020). Os avanços dos exames de imagem na atualidade levaram a um aumento na detecção precoce, sendo relevante a abordagem clínica, diagnóstica e terapêutica da DC (PASSOS ARO, et al., 2017).

A DC clássica, ocorre quando há grande envolvimento dos ductos biliares intra-hepáticos e se manifesta com colangite recorrente sem fibrose periportal. Na Síndrome de Caroli (SC), as dilatações císticas ocorrem em conjunto com a fibrose hepática congênita (LEE JM, et al., 2015). Essas anomalias estão associadas à expressão do gene autossômico recessivo da doença renal policística (ARPKD). Outras patologias também podem estar relacionadas a essa alteração genética como insuficiência renal, hipoplasia pulmonar e fibrose hepática (SWEENEY WE e AVNER ED, 2019).

A dilatação da árvore biliar predispõe à estase e formação de lama biliar que causam obstrução do fluxo. Dessa forma, os portadores dessa doença apresentam episódios recorrentes de colangite bacteriana, que se manifesta com dor abdominal, febre, calafrios e icterícia. As alterações da DC e SC também favorecem a ocorrência de hepatolitíase e cirrose biliar secundária à obstrução.

Pacientes com SC, em que há desenvolvimento de fibrose hepática, apresentam quadros como hipertensão portal, que pode levar à formação de varizes esofageanas, além de ascite e hepatomegalia (UMAR J, et al., 2020). Complicações como abscessos hepáticos e sepse podem comprometer o curso da doença. Além disso, estudos mostram uma frequência de 14 a 21\% de degeneração maligna em carcinoma colangiocelular (FAHRNER R, et al., 2019).

A história clínica detalhada, exame físico e de imagens são fundamentais para o diagnóstico da doença. Os exames laboratoriais apresentam resultados inespecíficos para DC e SC, mas podem retratar colestase e síndrome inflamatória aguda nos estágios iniciais (MOSLIM MA, et al., 2015). O diagnóstico é estabelecido por exames de imagem, sendo a ultrassonografia (US) mais utilizada e onde se observa a dilatação dos ductos biliares intra-hepáticos.

Na tomografia computadorizada (TC) pode ser observado o sinal do "ponto central" com imagem de dilatação cística escurecida com ponto central captando contraste. Outros exames como a colangiopancreatografia endoscópica retrógrada (CPRE) e a colangioressonância também podem evidenciar alterações (LEFERE M, et al., 2011; UMAR J, et al., 2020). 
As opções terapêuticas para essa doença são majoritariamente suportivas e devem ser indicadas de acordo com o grau de acometimento do paciente. O uso de ácido ursodesoxicólico pode ser usado em caso de colestase. A colangite bacteriana pode ser tratada com antimicrobianos e procedimentos cirúrgicos estão indicados em caso de obstrução ou para drenagem de abscessos.

Entre os tratamentos definitivos estão incluídos a ressecção hepática parcial, quando há acometimento localizado, e o transplante hepático, indicado quando há doença difusa concomitante à hipertensão portal sintomática (LAI Q e LERUT J, 2015).

O objetivo deste trabalho consiste em demonstrar, através de uma pesquisa bibliográfica, a importância do conhecimento da história clínica da Doença de Caroli, bem como elucidar as abordagens diagnósticas e terapêuticas desta patologia.

\section{REVISÃO BIBLIOGRÁFICA}

\section{Definição e epidemiologia}

A Doença de Caroli (DC), também classificada como cisto de colédoco do tipo V segundo a Classificação de Todani, é uma doença incomum, de caráter hereditário autossômico recessivo, em sua maioria, que compromete o trato biliar hepático e foi descrita pela primeira vez pelo médico francês Jacques Caroli, em 1958 (UNÇYÜREK Ö e LOMAS DJ, 2016; BAUMGARTNER K, et al., 2020). Sua origem se dá através de uma malformação na placa ductal que promove uma dilatação sacular, multifocal ou irregular sem caráter obstrutivo da árvore biliar intra-hepática (MOSLIM MA, et al., 2015; PASSOS ARO, et al., 2017; UMAR J, et al., 2020).

São mencionados 2 tipos de malformações de Caroli: Tipo I, também definido como doença de Caroli, e Tipo II, conhecido como Síndrome de Caroli (SC), caracterizado por ectasias císticas difusas (bilobar) do trato biliar intra-hepático, concomitante à presença de fibrose hepática congênita, hipertensão portal e doença renal (BAUMGARTNER K, et al., 2020; JARUFE N, et al., 2013). Ao mesmo tempo em que a Síndrome de Caroli, que é mais comum que a $\mathrm{DC}$, está mais relacionada à presença de fibrose hepática congênita associada, a DC está inserida em um conjunto de doenças consideradas fibropolicísticas hepáticas. Estas surgem de acordo com os vários estágios da pausa da placa ductal embriológica (DP), como exemplo, a fibrose hepática congênita, doença renal policística autossômica dominante (ADPKD) e cistos do colédoco (BAUMGARTNER K, et al., 2020; UNÇYÜREK Ö e LOMAS DJ, 2016).

A incidência e prevalência da DC ainda não são conhecidas de maneira fidedigna, justamente por ser uma doença rara, porém, em virtude da melhoria na imagem transversal e melhor compreensão da patologia, parece que tem ocorrido aumento na prevalência. No que tange a incidência, é estimado 1 caso para cada 1.000.000 de habitantes/ano, sendo homens e mulheres igualmente afetados, mais comum em descendentes asiáticos e com pico de incidência no início da idade adulta, sendo que mais de $80 \%$ dos pacientes manifestam a DC antes dos 30 anos de idade (MOSLIM MA, et al., 2015; UMAR J, et al., 2020).

\section{Fisiopatologia e manifestações clínicas e laboratoriais}

A Doença de Caroli, reconhecida pela deformidade do sistema biliar, tem sua patogênese iniciada ainda nas primeiras semanas de gestação. No ambiente intrauterino, ocorre uma má-formação da placa ductal, caracterizada pela persistência de estruturas biliares embrionárias, que desencadeia um desenvolvimento anormal das vias biliares (BAUMGARTNER K, et al., 2019; COURCET JB, et al., 2015). A gravidade da anomalia e o grau de comprometimento da árvore porto-biliar determinará o espectro de malformações, abrangendo fibrose hepática congênita, DC (ductos grandes e médios) e SC (ductos médios e microscópicos) (PASSOS ARO, et al., 2017).

A fisiopatologia completa dessa alteração ainda não é totalmente elucidada, mas já se sabe que ocorre uma mutação do gene PKHD1, localizado no cromossomo $6 \mathrm{p} 12$, que é mais evidente nos rins, embora também esteja presente no pâncreas e no fígado, em níveis inferiores. Tal gene codifica a proteína fibrocistina, cuja função é a manutenção da arquitetura tubular tridimensional, além da monitorização da multiplicação celular no desenvolvimento normal do fígado e dos rins (UMAR J, et al., 2020). 
Desse modo, a fibrocistina anormal ou ausente provoca uma falha estrutural no parênquima hepático, que tem como apresentação as dilatações saculares das vias biliares, que podem ser focais ou difusas (UNÇYÜREK Ö e LOMAS DJ, 2016; SRINATH A e SHNEIDER BL, 2012).

O quadro clínico dessa patologia surge com anomalias hepáticas e renais. As complicações renais, representadas por rins policísticos congênitos, se manifestam precocemente, devido à maior expressão do gene acometido da fibrocistina nas células dos túbulos renais, enquanto que as desordens hepáticas são apresentadas mais tardiamente (UMAR J, et al., 2020).

Clinicamente, a Doença de Caroli pode persistir assintomática nas primeiras duas décadas ou se manifestar de uma forma oligossintomática no decorrer da vida do paciente (PASSOS ARO, et al., 2017). Os sinais clínicos, quando presentes, são variados, sendo os traços de infecção os mais marcantes da doença. Estes decorrem do acúmulo de bile, que promove bloqueio da secreção biliar, ocasionando quadro repetidos de colangite bacteriana, podendo evoluir para formação de abcessos e até sepse (LENDOIRE JC, et al., 2011; UMAR J, et al., 2020).

A colangite é representada classicamente pela tríade de Charcot, caracterizada por icterícia, febre com calafrios e dor abdominal no quadrante superior direito. Devido ao aumento de bilirrubina plasmática, o prurido também pode compor o quadro (BAUMGARTNER K, et al., 2019; UMAR J, et al., 2020). É incomum a apresentação da doença em pacientes com idade avançada, após a instalação de fibrose hepática, quando surgem sinais e sintomas de hipertensão portal, tais como ascite e varizes (UMAR J, et al., 2020).

Ao exame físico, é possível observar a ocorrência de dor no hipocôndrio direito com sinal de Murphy negativo, além de icterícia e hepatomegalia. A presença de ascite e aranha vascular podem evidenciar 0 desenvolvimento de cirrose hepática. (UMAR J, et.al., 2020). Quanto à avaliação laboratorial, há evidência leucocitose. Os níveis elevados de bilirrubina direta e fosfatase alcalina convergem com um quadro de colangite. Geralmente, os marcadores de função hepática se mantém inalterados (UMAR J, et al., 2020).

Por fim, há relatos de casos crescentes de colelitíase intra-hepática, o que aumenta em 30 vezes o risco de desenvolvimento de colangiocarcinoma, definidamente o câncer mais comum das vias biliares e possuidor de um prognóstico desanimador (BAUMGARTNER K, et al.,2019; BLECHACZ B, 2017; UMAR J, et al., 2020).

\section{Diagnóstico e prognóstico}

O diagnóstico da Doença de Caroli e da Síndrome de Caroli consiste em demonstrar a comunicação entre os segmentos dilatados de ductos biliares intrahepáticos (IHBD) e do restante do sistema biliar (LEFERE M, et al., 2011). Para elucidar a confirmação, várias técnicas podem ser usadas, como o ultrassom (US), a tomografia computadorizada (TC) e a colangiopancreatografia por ressonância magnética (CPRM), sendo essa última muito significativa devido ao excelente contraste com o tecido, proporcionando maior precisão e visualização de achados esperados (BAUMGARTNER K, et al., 2020).

O US geralmente é o primeiro exame realizado e proporciona a visualização de dilatações saculares dos ductos intra-hepáticos. Já a TC, mostra um fígado dismórfico, com dilatações (UMAR J, et al., 2020). A presença de pequenos pontos de forte realce pelo contraste dentro dos ductos biliares intra-hepáticos dilatados na tomografia computadorizada (TC) ou ressonância magnética é considerada altamente específica para a Doença de Caroli (LEFERE M, et al., 2011).

Comumente é visto a expansão do ducto biliar e o "sinal de ponto central", que denota um feixe fibrovascular, consistindo em uma radícula da veia porta e um ramo da artéria hepática, rodeado por um ducto biliar aumentado. Dependendo do plano de secção, o feixe pode aparecer como um espessamento linear ou como um ponto. De acordo com a literatura, esse achado é altamente sugestivo dessa patologia (BAUMGARTNER K, et al., 2020).

Ademais, a CPRM é uma alternativa não invasiva para a colangiografia percutânea transhepática (CPT) ou colangiografia retrógrada endoscópica (CPRE), proporcionando boa adesão do paciente sem haver necessidade de métodos invasivos mais complicados. A vantagem da realização de CPRE é no quesito terapêutico, uma vez que pode ser removido cálculos por meio de esfincterotomia e varredura com o balão (UMAR J, et al., 2020). Ainda na CPRM, podem ser visualizadas outras condições patológicas, como cistos renais, pancreáticos, hepáticos e peribiliares (BAUMGARTNER K, et al., 2020). 
Além disso, a ressonância pode ser usada como única modalidade na avaliação diagnóstica de pacientes com suspeita de Doença de Caroli, principalmente quando apresentam dilatações saculares e sinais de pontos cerebrais (Figura 1 A e B) (LEFERE M, et al., 2011). A biópsia hepática raramente é realizada e, quando feita, possui indicação para buscar evidências de fibrose hepática (UMAR J, et al., 2020).

Os diagnósticos diferenciais incluem a Doença Policística do Fígado, Complexo de Von Meyenburg (microhamartomas biliares, caracterizados por dilatações císticas dos ductos biliares intra-hepáticos, imbuídas em grande quantidade de estroma fibroso) e tumores do ducto biliar, que podem causar colestase, colangite piogênica recorrente e metástases hepáticas (BAUMGARTNER K, et al., 2020; PECH L, et al., 2015).

Raramente a doença se manifesta em idades avançadas, sendo que o prognóstico da doença se modifica de acordo com a extensão do acometimento, da anormalidade genética - exclusão ou mutação de genes -, e com a quantidade de diferentes sistemas envolvidos. Por isso, a terapêutica deve se manter individualizada, evitando o avanço da comorbidade. Quando há obstrução biliar recorrente e fibrose, a morbidade se faz mais significativa, o que concede ao transplante de fígado o único tratamento definitivo. Aqueles pacientes que apresentarem colangiocarcinoma, irão evoluir com um pior prognóstico (UMAR J, et al., 2020).

Figuras 1 A e B: Colangiorressônancia evidenciando dilatação de vias biliares intra-hepáticas, compatível com Doença de Caroli.
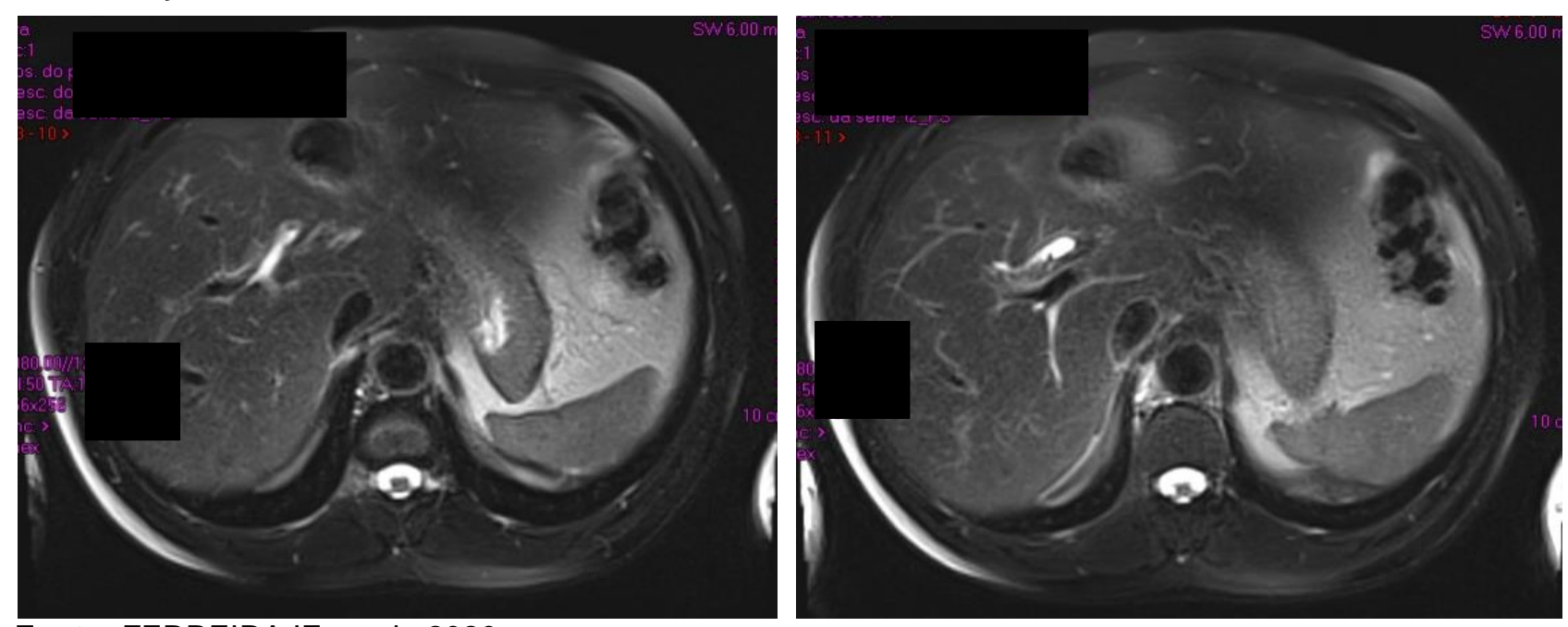

Fonte: FERREIRA IF, et al., 2020.

\section{Tratamento e gestão}

A base do tratamento é de suporte e a opção terapêutica de escolha varia de acordo com a extensão do segmento afetado pela Doença de Caroli (UMAR J, et al., 2020; ALVANOS A, et al., 2019). A resolução das dilatações císticas da via biliar se dá, basicamente, por intervenção cirúrgica, que se baseia na sua classificação e na existência de associação com doença hepática, sendo o objetivo principal a eliminação completa dos cálculos intra-hepáticos e a prevenção de episódios recorrentes de colangite e, posteriormente, de fibrose hepática e colangiocarcinoma secundário (PACHECO EG, et al., 2015; CLEMENTE G, et al., 2010; JARUFE N, et al., 2012).

Quando a doença intra-hepática é unilobar, localizada e não possui associação com doença hepática crônica, a ressecção hepática consiste no procedimento de escolha, apresentando desfechos excelentes à longo prazo. Em contrapartida, quando a doença acomete ambos os lobos do fígado ou está associada a alguma patologia hepática subjacente, como hipertensão portal, fibrose hepática e cirrose biliar secundária, os resultados após tratamento conservador não são satisfatórios (MABRUT JY, et al., 2010).

A colangite, apresentação bastante comum da Doença de Caroli, é tratada com antibióticos de amplo espectro e boa penetração na via biliar, como as cefalosporinas de $3^{\underline{a}}$ geração e as quinolonas, objetivando a cobertura de germes gram-negativos e anaeróbios, responsáveis em grande parte pela etiologia desta condição. 
Na necessidade de uma terapia adjunta, pode-se utilizar-se da drenagem biliar por meio da colocação de stent biliar através de Colangiopancreatografia Retrógrada Endoscópica (CPRE) ou de cateter trans-hepático percutâneo (PTC). O PTC geralmente apresenta maior eficácia na desobstrução intra-hepática e os pacientes podem necessitar de cateteres de demora com irrigação periódica e troca dos mesmos. Nos casos de colestase grave, há indicação para o uso de ácido ursodeoxicólico (UMAR J, et al., 2020; PACHECO EG, et al., 2015).

Se instalada, a fibrose hepática culmina em hipertensão portal e, consequentemente, podem ocorrer episódios de sangramentos decorrentes de varizes e ascite recorrente, que devem receber o mesmo tratamento de quando estão quando associadas à cirrose hepática: beta-bloqueadores não seletivos e ligadura elástica endoscópica para varizes, e diurético para os casos de ascite (UMAR J, et al., 2020).

O transplante hepático constitui o último recurso terapêutico de sucesso para as formas graves da doença e fornece resultados excepcionais à longo prazo. Possui 3 indicações específicas para a sua realização, sendo (1) descompensação hepática, (2) colangite recorrente refratária ao tratamento e (3) evolução para adenocarcinoma focal. Como as chances do desenvolvimento de complicações sépticas e do surgimento do colangiocarcinoma aumentam com o tempo, a intervenção cirúrgica oportuna é de fundamental importância, visto o reservado prognóstico apresentado por estas situações (UMAR J, et al., 2020; FAHRNER R, et al., 2018).

$\mathrm{Na}$ ocorrência de colangiocarcinoma, ainda que a intervenção cirúrgica seja considerada a única opção potencialmente curativa, as taxas de recorrência após a ressecção ainda são significativamente elevadas (49 a 64\%), de localização majoritariamente intra-hepática e com ocorrência de cerca de 2 a 3 anos após o procedimento. Além disso, como a maior parte dos pacientes com essa neoplasia são diagnosticados tardiamente, 10 a $45 \%$ daqueles considerados ressecáveis, descobrem ser irressecáveis durante a realização da laparotomia exploratória, fato este que contribui ainda mais para um prognóstico desfavorável (BLECHACZ B, 2017).

Nos casos em que ocorre acometimento concomitante dos rins e do fígado, o transplante combinado de rim e fígado (CKLT) simultâneo (melhor) ou sequencial deve ser idealizado considerando as respectivas evoluções das doenças renais e hepáticas. Como o número de casos relatados ainda é restrito, ainda não é possível estabelecer um consenso sobre o melhor método terapêutico (transplante hepatorrenal único ou combinado - simultâneo ou sequencial) e nem sobre qual a melhor circunstância para que essa intervenção ocorra.

É importante considerar o fato de que o aloenxerto hepático oferece imunoproteção ao enxerto renal se o transplante ocorrer sincronicamente (identidade imunogenética), mas supostamente, o mesmo não ocorre em rins transplantados posteriormente. Tal indício parece ser um forte fundamento em prol da realização de um transplante hepatorrenal simultâneo (LAI Q e LERUT J, 2016).

\section{Complicações}

A despeito de uma terapêutica inicial apropriada, o seguimento de pacientes portadores de Doença de Caroli pode ser afetado por intercorrências variadas, à longo prazo. As complicações mais comuns podem ser referentes tanto àquelas gerais, decorrentes de cirurgias abdominais, quanto àquelas mais específicas, consequentes ao manejo das vias biliares. Entre as complicações precoces, as fístulas biliares são consideradas as mais temidas e, em grande parte dos casos, podem ser resolvidas com tratamento conservador, incluindo drenagem e direcionamento da bile, além de, ocasionalmente, ser necessária a associação da somatostatina para a resolução do quadro (MABRUT JY, et al., 2010; PACHECO EG, et al., 2015).

Ainda nessa classificação, é possível abranger outras intercorrências, como abscesso de parede e abscessos hepáticos, flebites, insuficiência renal e hepática (quando há ressecção associada), além de sangramentos de anastomose biliodigestiva. Com relação às complicações pós-operatórias tardias, pode-se citar litíase biliar, especialmente na forma de coledocolitíase, colangite, hérnia incisional e estenose intrahepática parcial ou completa. 
Estudos afirmam que alguns fatores de risco podem influenciar para o desenvolvimento da estenose anastomótica, como o tipo de cisto das vias biliares, o aumento do tamanho desse cisto, a duração breve dos sintomas e o estágio de infiltração de células inflamatórias (MABRUT JY, et al., 2010; PACHECO EG, et al., 2015).

Diante disso, embora seja uma enfermidade rara, a Doença de Caroli pode gerar diversas intercorrências no paciente, que variam em grau de severidade e, portanto, necessitam de um acompanhamento multidisciplinar, em que a equipe seja composta por gastroenterologista, geneticista, radiologista, nefrologista, urologista e cirurgião. Assim, é possível não só estabelecer um completo planejamento diagnóstico e terapêutico, como também aumentar as chances de precaver possíveis complicações (PACHECO EG, et al., 2015; UMAR J, et al., 2020).

\section{CONSIDERAÇÕES FINAIS}

A Doença de Caroli, apesar de considerada rara, pode afetar significativamente a qualidade de vida do paciente, devido às repercussões sistêmicas advindas de quadros infecciosos (colangite, abscesso e sepse), e de suas possíveis complicações, dentre as quais pode-se citar fibrose hepática, hipertensão portal e colangiocarcinoma. Devido à sua gravidade e, consequentemente, elevada morbimortalidade, o diagnóstico precoce se torna imprescindível, sendo, portanto, fundamental o conhecimento do profissional da saúde acerca dos sinais e sintomas dessa patologia. Posteriormente, faz-se necessário individualizar o tratamento de acordo com a extensão e comprometimento da doença, além de estabelecer uma boa relação médicopaciente, tendo em vista que esses pacientes necessitarão ser acompanhados por toda a vida.

\section{REFERÊNCIAS}

1. ALVANOS A, et al. Abordagem Cirúrgica com benigno Alterações do ducto biliar. O cirurgião, 2019; 91: 11-17.

2. BAUMGARTNER, K, et al. Caroli-Syndrom. RöFo-Fortschritte auf dem Gebiet der Röntgenstrahlen und der bildgebenden Verfahren, 2020; 192: 119-122.

3. BLECHACZ B. Colangiocarcinoma: Conhecimento Atual e Novos Desenvolvimentos.Gut and Liver, 2017 ; 11 (1): 1326.

4. CLEMENTE G, et al. Liver resection for intrahepatic stones in congenital bile duct dilatation. Journal of Visceral Surgery, 2010; 147(3): 175-180.

5. COURCET JB, et al. Composto Heterozigoto PKHD1 Variantes causam um amplo espectro de malformações da placa ductal. American Journal of Medical Genetics, 2015; 167A: 3046-3053.

6. JARUFE NC, et al. Definições e conceitos em hepatolitíase. Revista Chilena de Cirugía, 2013; 65(4): 360-363.

7. JARUFE N, et al. Hepatectomia anatômica como tratamento definitivo para hepatolitíase: um estudo de coorte. HPB, 2012; 14(9): 604-610.

8. LAI Q e LERUTE J. Proposta de algoritmo para transplante de fígado na doença e síndrome de Caroli: colocando um esforço incomum em uma tarefa comum. Clinical Transplantation, 2016; 30: 3-9.

9. LEE JM, et al. Nephronophthisis 13: implications of its association with Caroli disease and altered intracellular localization of WDR19 in the kidney. Pediatric Nephrology, 2015; 30(9): 1451-1458.

10. LENDOIRE JC, et al. Doença de Caroli: relato de opções cirúrgicas e resultados em longo prazo de pacientes tratados na Argentina. Estudo Multicêntrico. Journal of Gastrointestinal Surgery, 2011; 15: 1812-1819.

11. MABRUT JY, et al. Tratamento de cistos do ducto biliar congênito. Digestive Surgery, 2010; 27: 12-18.

12. MOSLIM MA, et al. Tratamento Cirúrgico de Caroli ' Doença: Experiência em um único centro e revisão da literatura. Journal of Gastrointestinal Surgery, 2015; 19(11): 2019-2027.

13. PACHECO EG, et al. Doenças císticas das vias biliares. Revista da Sociedade Brasileira de Clínica Médica, 2015; 13(3): 213-217.

14. PASSOS ARO, et al. Doença de Caroli: revisão de literatura. Brazilian Journal of Surgery and Clinical Research, 2018; 21(1): 103-105.

15. PECH L, et al. Imagem dos complexos de Von Meyenburg. Diagnostic and Interventional Imaging, 2015; 97(4): 401409.

16. PLENTZ RR, MALEK NP. Apresentação clínica, fatores de risco e sistemas de estadiamento de colangiocarcinoma. Melhores práticas e pesquisas em gastroenterologia clínica, 2015; 29(2): 245-252.

17. SRINATH A, SHNEIDER BL. Fibrose Hepática Congênita e Recessiva Autossômica Doença renal policística. Journal of Pediatric Gastroenterology and Nutrition, 2012; 54(5): 580-587.

18. SWEENEY WE, AVNER ED. Polycystic Kidney Disease, Autosomal Recessive. Gene Reviews, 2019; 1-26.

19. TUNÇÜREK Ö, LOMAS DJ. A doença de Caroli deve estar na classificação de Todani? Springer Science + Business Media New York, 2016, 41:1-2.

20. UMAR J, et al. Doença de Caroli. NCBI Bookshelf, 2020; 2-5. 